\title{
Ocorrência de Bruquíneos (Coleoptera: Bruchidae) em Sementes de Emmotum nitens
}

\author{
(Benth.) Miers \\ Occurrence of Bruquine (Coleoptera: Bruchidae) in Seeds of Emmotum nitens (Benth.) Miers \\ Presencia de Brucines (Coleoptera: Bruchidae) en semillas de Emmotum nitens (Benth.) Miers
}

Recebido: 01/09/2021 | Revisado: 08/09/2021 | Aceito: 09/09/2021 | Publicado: 12/09/2021

\author{
Marcus Vinicius Prado Alves \\ ORCID: https://orcid.org/0000-0001-5579-8011 \\ Universidade Federal de Lavras, Brasil \\ E-mail: ordapsevla@yahoo.com.br \\ Jose Carlos Sousa Silva \\ ORCID: https://orcid.org/0000-0001-5648-6773 \\ Empresa Brasileira de Pesquisa Agropecuária Cerrados, Brasil \\ E-mail: jose.sousa-silva@embrapa.br \\ Cassia Duarte Oliveira \\ ORCID: https://orcid.org/0000-0002-2748-2930 \\ Universidade Federal de Lavras, Brasil \\ E-mail: cassiaduartemg2@hotmail.com \\ Antonia Isadora Fernandes \\ ORCID: https://orcid.org/0000-0001-7101-562X \\ Universidade Federal de Lavras, Brasil \\ E-mail: fernandesisadora_@hotmail.com
}

\begin{abstract}
Resumo
Emmotum nitens (Benth.) Miers, conhecida popularmente como Sobre, Carvalho-do-Cerrado, Salgueiro, entre outros, pertence à família Icacinaceae, está amplamente dispersa pelos Cerrados do Brasil, sendo uma das espécies de maior importância. Os coleópteros da subfamília Bruchidae (Chrysomelidae) são importantes predadores de sementes, principalmente de leguminosas, sendo que suas larvas se alimentam exclusivamente de sementes, atacando principalmente o embrião. O objetivo neste trabalho foi elucidar a oviposição, desenvolvimento e ciclo completo de coleópteros da subfamília Bruchidae em frutos e sementes de E. nitens. Foi observada uma porcentagem de $47 \%$ de frutos com a presença de oviposição na face externa (exocarpo) e 21,4\% de sementes predadas por estes bruquíneos. Após o seu ciclo completo, os coleópteros adultos emergem pelo orifício presente na parede do mesocarpo. O tempo de seu ciclo e seus instares levam em torno de seis meses.
\end{abstract}

Palavras-chave: Coleópteros; Diásporos; Icacinaceae.

\begin{abstract}
Emmotum nitens (Benth.) Miers, popularly known as Sobre, Carvalho-do-Cerrado, Salgueiro, among others, belongs to the family Icacinaceae, is widely dispersed in the Cerrados of Brazil, being one of the most important species. The beetles of the subfamily Bruchidae (Chrysomelidae) are important seed predators, mainly of legumes, and their larvae feed exclusively on seeds, mainly attacking the embryo. the objective of this work was to elucidate the oviposition, development and complete cycle of beetles of the subfamily Bruchidae in fruits and seeds of E. nitens. A percentage of $47 \%$ of fruits with the presence of oviposition on the external face (exocarp) and $21.4 \%$ of seeds predated by these bruchines were observed. After their complete cycle, adult beetles emerge through the hole present in the wall of the mesocarp. Its cycle time and urges take around six months.
\end{abstract}

Keywords: Coleoptera; Diaspores; Icacinaceae.

\section{Resumen}

Emmotum nitens (Benth.) Miers, popularmente conocido como Sobre, Carvalho-do-Cerrado, Salgueiro, entre otros, pertenece a la familia Icacinaceae, se encuentra ampliamente disperso en los Cerrados de Brasil, siendo una de las especies más importantes. Los escarabajos de la subfamilia Bruchidae (Chrysomelidae) son importantes depredadores de semillas, especialmente de leguminosas, y sus larvas se alimentan exclusivamente de semillas, atacando principalmente al embrión. El objetivo de este trabajo fue dilucidar la oviposición, desarrollo y ciclo completo de escarabajos de la subfamilia Bruchidae en frutos y semillas de E. nitens. Hubo un porcentaje de $47 \%$ de frutos con presencia de oviposición en la cara externa (exocarpio) y 21,4\% de semillas antes de estas bruchinas. Después de su ciclo completo, los escarabajos adultos emergen a través del orificio en la pared del mesocarpio. Tu ciclo e impulsos tardan unos seis meses.

Palabras clave: Coleoptera; Diásporas; Icacinaceae. 


\section{Introdução}

Emmotum nitens (Benth.) Miers pertence à família Icacinaceae, alcança até $10 \mathrm{~m}$ de altura, e está amplamente dispersa pelos Cerrados do Brasil, sendo uma das espécies de maior índice de importância, Ribeiro et al., (1985); Felfili et al., (2002); Ribeiro \&Walter, (2008). Conhecida popularmente como Sobre, Carvalho-do-Cerrado, Faia, Pau-de-Sobre, Salgueiro, entre outros (Almeida et al., 1998). De acordo com Alves e Silva (2013) o desenvolvimento dos frutos é lento, ocorrendo por um longo período (abril - setembro). A dispersão dos frutos ocorre por barocoria entre os meses de outubro a janeiro. Os frutos na época de maturação e dispersão apresentam-se com colorações verdes e marrom-escuras (Alves \& Silva, 2013).

A espécie possui importância econômica e ecológica para construções rurais e recuperação de áreas degradadas. Seus frutos são do tipo carnoso, com mesocarpos lenhosos e sua dispersão ocorre durante a estação chuvosa por barocoria (Almeida et al., 1998); Moreira (1987); Fernandes-Bulhão (2002)

Os coleópteros da subfamília Bruchidae (Chrysomelidae) são importantes predadores de sementes, principalmente de leguminosas, sendo que suas larvas se alimentam exclusivamente de sementes, atacando principalmente o embrião, e devido a este fato, algumas espécies desta subfamília são consideradas grandes pragas, pois as sementes de algumas espécies são de grande importância ecológica, econômica ou utilizada na alimentação humana (Romero-Napoles 2002; Kingsolver 2004).

Pouco se conhece a respeito do desenvolvimento dos bruquíneos que consomem espécies florestais, já que o estudo de seu ciclo biológico é bastante dificultado pelo fato de suas larvas se desenvolverem no interior das sementes. Sabe-se que na fase adulta, os bruquíneos possuem basicamente apenas função reprodutiva, alimentando-se apenas de pólen, néctar ou não se alimentam (Romero-Napoles 2002; Kingsolver 2004).

Apesar de toda a sua importância e características, não foram encontrados na literatura estudos relacionados à predação de sementes de Emmotum nitens e sua relação com a reprodução e estabelecimento. Assim, o objetivo neste trabalho foi elucidar a oviposição, desenvolvimento e predação de sementes e o ciclo completo de coleópteros da subfamília Bruchidae (Chrysomelidae) em frutos e sementes de Emmotum nitens (Benth.) Miers.

\section{Metodologia}

O estudo foi realizado com 15 matrizes da espécie Emmotum nitens, entre outubro de 2010 a dezembro de 2011, na área da Embrapa Cerrados - Centro de Pesquisa Agropecuária dos Cerrados - CPAC, mais especificamente nas proximidades da Reserva Ecológica Cerradão, Planaltina - Distrito Federal situada a $35 \mathrm{~km}$ de Brasília, nas coordenadas (15²35' 30’' S e $47^{\circ} 42^{\prime} 30^{\prime}$ 'W) com aproximadamente 998 metros de altitude.

Os frutos de Emmotum nitens foram coletados manualmente com o auxílio de sombrites estendidos sob a copa de quinze indivíduos adultos, entre 15 de outubro e 15 de dezembro de 2010 (período chuvoso). Após cada coleta, os frutos foram levados ao Laboratório de Biologia Vegetal da Embrapa Cerrados.

O beneficiamento dos frutos de E. nitens consistiu na remoção do exocarpo e consequente obtenção dos mesocarpos. O mesocarpo foi considerado como "diásporo", uma vez que esta estrutura abriga o embrião.

Os mesocarpos após o beneficiamento foram homogeneizados, e então acondicionados em sacos de papel $(50$ x $35 \mathrm{~cm}$ / 0,25 mm), sendo armazenados a temperatura ambiente do Laboratório de Biologia Vegetal da Embrapa Cerrados - TA, a 25 ${ }^{\circ} \mathrm{C}\left( \pm 2{ }^{\circ} \mathrm{C}\right)$ e $60 \%( \pm 3 \% \mathrm{UR})$.

Por meio da constatação da presença de coleópteros adultos emergindo do interior dos mesocarpos (n=1000) armazenados em temperatura ambiente, e, a presença de larvas, pupas e coleópteros adultos em sementes de Emmotum nitens, foram, então, realizadas observações mais detalhadas em relação ao ciclo destes coleópteros, com o auxílio de um estéreo microscópio binocular (Carls Zeiss - 10x). 


\section{Resultados e Discussão}

\subsection{Presença de oviposição e sementes predadas por bruquíneos}

Foi observada uma porcentagem de $47 \%$ de frutos com a presença de oviposição na face externa (exocarpo) dos frutos e 21,4\% de sementes predadas por estes bruquíneos. As larvas e adultos destes coleópteros foram identificados como sendo da família (Bruchidae) através de literatura (Ribeiro \& Almeida, 2009), e confirmada no Laboratório de Entomologia da Embrapa Cerrados.

As relações da infestação de insetos nas estruturas reprodutivas podem estar correlacionadas com a fase de desenvolvimento, como pode ser percebido em alguns bruquídeos que preferem colocar seus ovos em frutos de Ipomoea pescaprae, em fase de amadurecimento (Castelanni \& Santos, 2005). Todavia, alguns insetos não apresentam essa preferência, como no caso de curculionídeos que atacam frutos jovens e maduros de Ocotea porosa (Cuaranhua, 2010).

Em outros casos, essas interações entre insetos e estruturas reprodutivas podem estar ligadas as características físicas, como consistência e o tamanho dos frutos que podem atrair determinados insetos, como as fêmeas de bruquídeos, que realizam as posturas no início do desenvolvimento dos frutos, acreditando-se que isto pode facilitar a entrada das larvas, devido a consistência do fruto (Grenha et al.,2008), porém, várias espécies de bruquídeos apresentam preferências por sementes maiores (Lomônaco,1994).

Estas informações foram de caráter preliminar, sendo ainda preciso realizar estudos mais aprofundados. Os diásporos $(\mathrm{n}=1000)$ apresentaram uma porcentagem de $21,4 \%$ de sementes predadas por coleópteros da família Bruchidae, num total de 1000 diásporos observados na Figura 1.

Figura 1. Número de sementes predadas (em porcentagem) por coleópteros (Bruchidae) em dez repetições de 100 sementes de diásporos (mesocarpos) armazenados em temperatura ambiente.

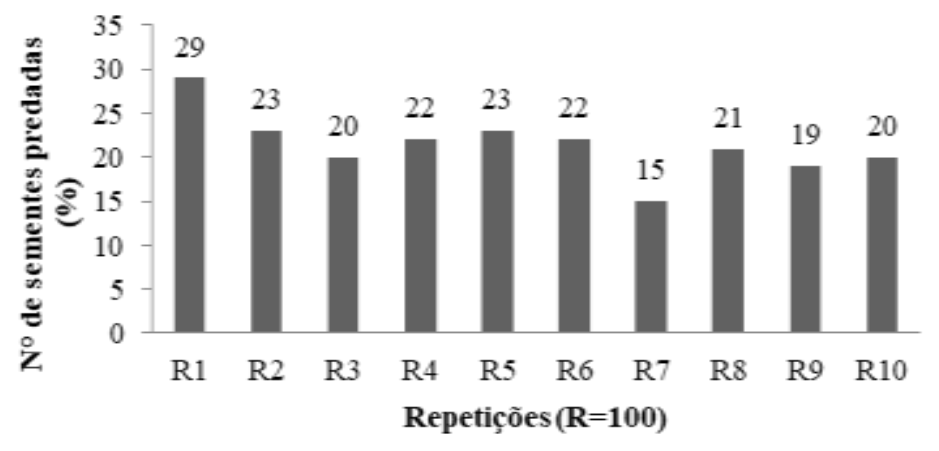

Fonte: Autores (2021).

\subsection{Ciclo de Bruchidae observado em frutos de Emmotum nitens}

A oviposição é feita no exocarpo (polpa), pelo adulto em frutos jovens de E. nitens, e com o mesocarpo ainda não lignificado. Após a eclosão, a larva penetra o fruto se estabelecendo em um dos lóculos; onde então, as larvas se desenvolvem no interior dos lóculos, passando pelas fases de pupa, ninfa, até se tornarem insetos adultos, sendo que durante o seu desenvolvimento, estas se alimentam da semente. Ao final do desenvolvimento da larva, esta, perfura o mesocarpo.

O comportamento de oviposição em frutos no campo ainda em fase de desenvolvimento por bruquídeos também foram constatadas por Santos et al., (1994), Kageyama \& Piña-Rodrigues (1993), Ribeiro-Costa \& Costa (2002) e Sari.et al (2005). De acordo com Sari.et al (2005) a ovoposição provavelmente ocorre neste período pela textura do fruto ser mais macia, o que facilita a entrada da larva, uma vez que larvas são incapazes de penetrar na parede do fruto quando estes estão parcialmente ou completamente lignificados. 
A preferência de bruquídeos por determinados sítios de oviposição pode ser uma maneira para sobrepor as barreiras impostas pela planta hospedeira, ou mesmo uma estratégia para prevenir a mortalidade de seus ovos pela ação de inimigos naturais na superfície dos frutos (Center \& Jonhson, 1974; Ribeiro-Costa \& Costa, 2002; Sari.et al, 2005).

Após o seu ciclo completo, os coleópteros adultos emergem pelo orifício presente na parede do mesocarpo. O tempo de seu ciclo e seus instares levaram em torno de seis meses. Tempo este que coincidiu com o início da frutificação de frutos de Emmotum nitens, no mês de abril; crescimento, maturação e dispersão dos frutos, em outubro de 2011 (Figura 2: A-I).

Figura 2. A - I. $A$ = Oviposição; $B=$ Larva no interior do lóculo; $C=$ Projeção da larva; $D=$ Semente parcialmente consumida pela larva; E - F = Perfuração na parede interior do mesocarpo feita pela larva; $G=$ Ninfa; $G=$ Mesocarpo perfurado; $\mathrm{H}$ = Coleóptero emergente adulto (Bruchidae).

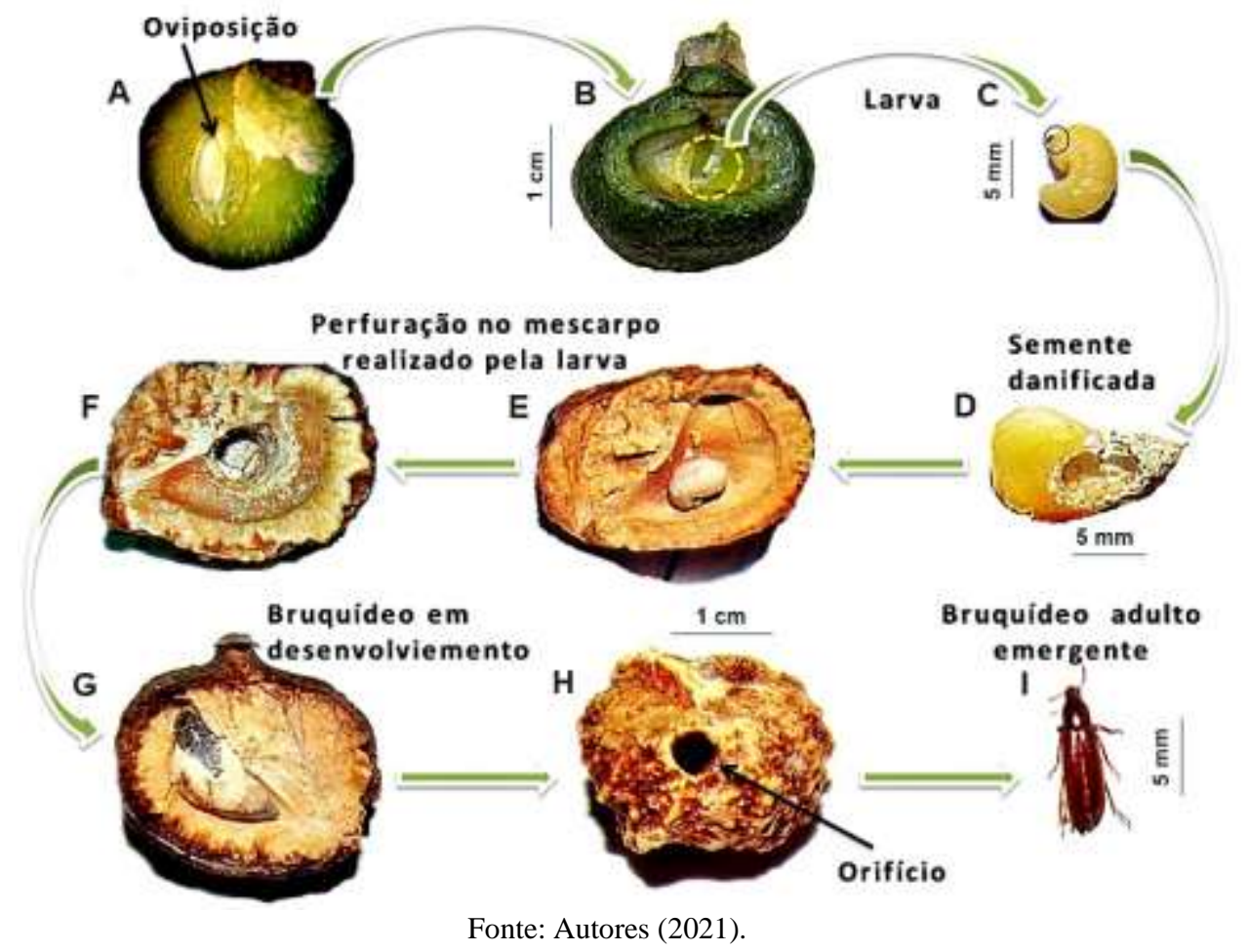

Arruda et al. (2015), avaliando a germinação de sementes de Acacia polyphylla em gerbox e papel como substrato, e observaram que $20 \%$ das sementes foram atacadas por bruquíneos. De acordo com Fonseca et al. (2020), o ataque de bruquíneos reduziu a germinação das sementes de Senegalia polyphyllao, sendo essa redução de $73 \%$ na germinação de sementes atacadas por bruquíneos.

\section{Considerações Finais}

A oviposição por bruquíneos é feita em frutos ainda jovens, sendo que durante o desenvolvimento dos coleópteros, estes se alimentam das sementes. Contudo, são necessários estudos mais aprofundados sobre a predação de sementes por estes bruquíneos, para que se possa ter uma melhor compreensão da relação deste coleóptero e a espécie Emmotum nitens. Desta maneira, será possível elucidar qual o real dano dos bruquíneos às sementes, além do desenvolvimento e estabelecimento de plântulas. Pois a espécie Emmotum nitens se destaca nos levantamentos florísticos, como uma das espécies de maior índice de importância, principalmente em fitofisionomias de Cerradão. 


\section{Agradecimentos}

Universidade de Brasilia (UnB), Embrapa Cerrados, Coordenação de Aperfeiçoamento de Pessoal de Nível Superior (CAPES), Conselho Nacional de Desenvolvimento Científico e Tecnológico (CNPq).

\section{Referências}

Almeida, S. D., Proença, C. E., Sano, S. M., \& Ribeiro, J. F. (1998). Cerrado: espécies vegetais úteis. Embrapa-CPAC, 464.

Alves, M. V. P., \& Silva, J. C. (2013). Fenologia de Emmotum nitens, Benth., Miers, Icacinaceae, na Reserva Ecológica Cerradão, Embrapa Cerrados, Planaltina, DF. Revista Verde de Agroecologia e Desenvolvimento Sustentável, 8(1), 18.

Arruda, D. M., Brandão, D. O., Veloso, M. D. D. M., \& Nunes, Y. R. F. (2015). Germinação de sementes de três espécies de Fabaceae típicas de floresta estacional decidual. Pesquisa Florestal Brasileira, 35(82), 135-142.

Brasil. Ministério da Agricultura, Pecuária e Abastecimento. Secretaria de Defesa Agropecuária. (2009). Regras para análise de sementes.

Castellani, T. T., \& Santos, F. A. (2005). Fatores de risco à produção de sementes de Ipomoea pes-caprae. Brazilian Journal of Botany, $28,773-783$.

Center, T. D., \& Johnson, C. D. (1974). Coevolution of some seed beetles (Coleoptera: Bruchidae) and their hosts. Ecology, 55(5), 1096-1103.

Cuaranhua, C. J. (2010). Frutificação, dispersão e predação por insetos de frutos/sementes de imbuia (Ocotea porosa).

Felfini, J. M., Fagg, C. W., da SILVA, J. C. S., de Oliveira, E. C. L., Pinto, J. R. R., \& Ramos, K. M. O. (2002). Plantas da APA Gama e Cabeça de Veado: espécies, ecossistemas e recuperação. UNB. Departamento de Engenharia Florestal.

Fernandes-Bulhão, C. Padrões fenológicos de espécies arbóreas do Cerradão Distrófico na Reserva Ecológica da Embrapa cerrados. 2002. p-60 (Doctoral dissertation, Dissertação de Mestrado. Universidade de Brasília, Planaltina-DF).

Fonseca, A. G., Júnior, S. L. A., Titon, M., Lemes, P. G., \& Machado, E. L. M. (2020). Sementes de Senegalia polyphylla (Fabaceae) atacadas por bruquíneos podem ser viáveis? Pesquisa Florestal Brasileira, 40.

Grenha, V, Macedo, M. V, Monteiro, R. F. (2008). Predação de sementes de Allagoptera arenaria (Gomes) O’ Kuntze (Arecacea) por Pachymerus nucleorum Fabricium (Coleoptera, Crysomelidae, Bruchinae). Revista Brasileira da Entomologia, 52(1), 50-56.

Kageyama, P.Y. \& Pinã-Rodrigues, F. C. M. (1993). Fatores que afetam a produção de sementes. In: Aguiar, I. B., Pinã-Rodrigues, F. C. M., Figliolia, M. B. (Coord.). Sementes Florestais Tropicais. ABRATES, 19-46.

Kingsolver, J. M. (2004). Handbook of the Bruchidae of the United States and Canada (Insecta, Coleoptera).

Lomônaco, C. (1994). Predação de sementes de leguminosas por bruquídeos (Insecta: Coleoptera) na Serra dos Carajás, Pará, Brasil. Acta Botanica Brasilica, $8,121-127$.

Moreira, A. G. (1987). Aspectos demograficos de emmotum nitens (Benth) Miers (icacinaceae) em um cerradão distrofico no Distrito Federal.

Ribeiro, J. F., \& Walter, B. M. T. (2008). As principais fitofisionomias do bioma Cerrado. Cerrado: ecologia e flora, 1, $151-212$.

Ribeiro, J. F., Silva, J. C. S., \& Batmanian, G. J. (1985). Fitossociologia de tipos fisionômicos de cerrado em Planaltina, DF. Revista brasileira de botânica, $8(2)$.

Ribeiro-Costa, C.S., Almeida, L.M. (2009). Bruchidae (Coleoptera: Chrysomelidae). In: Panizzi, A. \& ParraJ.R.P. Bioecologia e Nutrição de Insetos: Base para o manejo Integrado de Pragas. Brasília: Embrapa Informação Tecnológica, Brasil.

Ribeiro-Costa, C. S., \& Costa, A. D. S. (2002). Comportamento de oviposição de Bruchidae (Coleoptera) predadores de sementes de Cassia leptophylla Vogel (Caesalpinaceae), morfologia dos ovos e descrição de uma nova espécie. Revista Brasileira de Zoologia, 19, $305-316$.

Romero Nápoles, J., Ayers, T. J., \& Dan Johnson, C. (2002). Cladistics, bruchids and host plants: evolutionary interactions in Amblycerus (Coleoptera: Bruchidae). Acta zoológica mexicana, (86), 01-16.

Santos, G. P., Araújo, F. D. S., Monteiro, A. J., \& Neto, H. F. (2015). Danos Causados por Plocetes SP. (Coleoptera, Curculionidae) e Lendoptera em Sementes de Guiné-do-Mato-Coutareae Hexandra (RUBIACEAE). Ceres, 41(238).

Sari, L. T., Ribeiro-Costa, C. S., \& Medeiros, A. C. S. (2002). Insects associated with seeds of Lonchocarpus muehlbergianus hassl. (Fabaceae) in Três Barras, Paraná, Brazil. Neotropical Entomology, 31, 483-486. 